\title{
MOULD LEVEL CONTROL FOR THE CONTINUOUS STEEL CASTING
}

\author{
Lubomír Smutný*, Radim Farana*, Antonín Víteček*, Dalibor Kačmář̌** \\ *VSB-Technical University of Ostrava, Department of Control Systems and Instrumentation, \\ 17. listopadu 15, Ostrava-Poruba, 708 33, Czech Republic \\ **Microsoft s.r.o. Prague, Czech Republic
}

\begin{abstract}
Existing systems for the control of mould level of continuous steel casting in Czech Steelworks have had problems with the stability of the steel level position. A new control algorithm is designed on the basis of mathematical modelling and digital simulation. This should better satisfy the actual demand on the control quality. A PI controller with a variable gain and dither signal was used. During several months of practical tests and the experimental tuning of the controller, parameters were determined regarding the required shape time diagram of the steel level for all types of steels, resulting in very good slab surface quality. Copyright $\mathbb{C} 2005$ IFAC
\end{abstract}

Keywords: non-linear control system, technology, robust control, steel manufacture.

\section{INTRODUCTION}

Control engineering and its application to industrial plants covers a wide application area and it exploits innovative approaches and design methods (Goodwin, et al., 2001). Continuous casting processes of slabs or round billets is a real time industrial process in which the casting products are continuously produced from molten steel. The molten steel is poured into a mould through a ladle and a tundish, and the steel surface layer is solidified. The slab is extracted from the mould by a pinch roll, completely solidified by cooling water, cut to a specified length by a cutter and forwarded to the following process.

The term "controlled pouring" includes a large number of process-oriented control tasks and adaptation controller tasks, supervisory SCADA/MMI (Jin-Luh Su, et al., 1994), which for example, allow casting to be started automatically and enhance the reliability of casting process (Kačmář 1997). The mould steel level control is only a small part of the control system but it is very important. Especially, the mould bath level control has a great effect on the surface quality of cast slab and billets and yield, so that the fluctuation of steel level position has to be closely controlled, as it is an important factor in process control (Böcher, et al., 1993). Fig. 1 shows the technological scheme of mould steel level control.

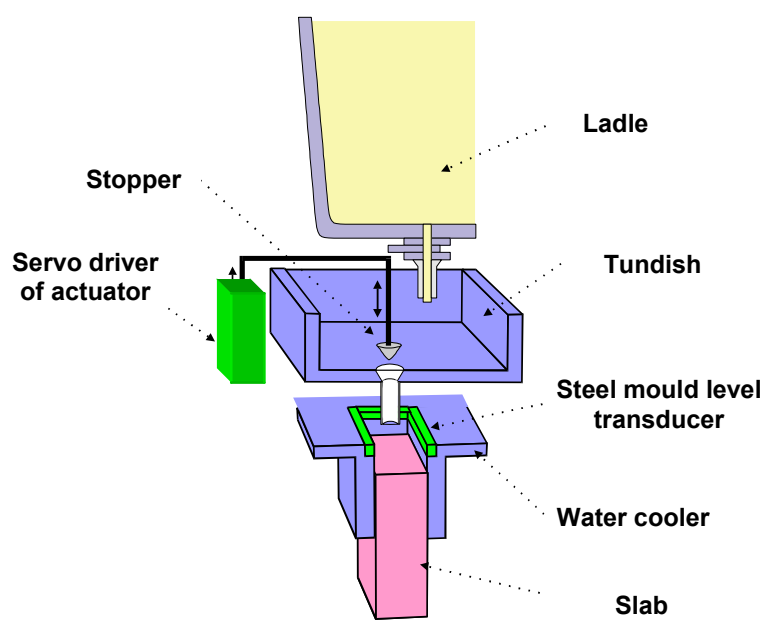

Fig. 1. Technological scheme of mould steel level control 


\section{PROCESS CONTROL ALGORITHMS}

One of the main tasks of the control system is to keep the level of molten metal in the mould constant. In principle, two procedures are employed, varying the flow of liquid metal from the tundish to the mould or varying the speed at which the strand is withdrawn (see Fig. 2). In case of high quality requirements, the input flow control is preferred. A mould steel level control system consists of several parts.

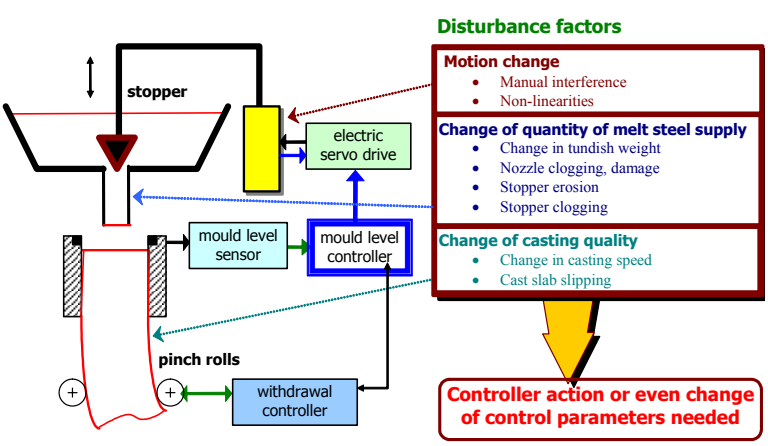

Fig. 2. Mould level control system and the disturbance factors

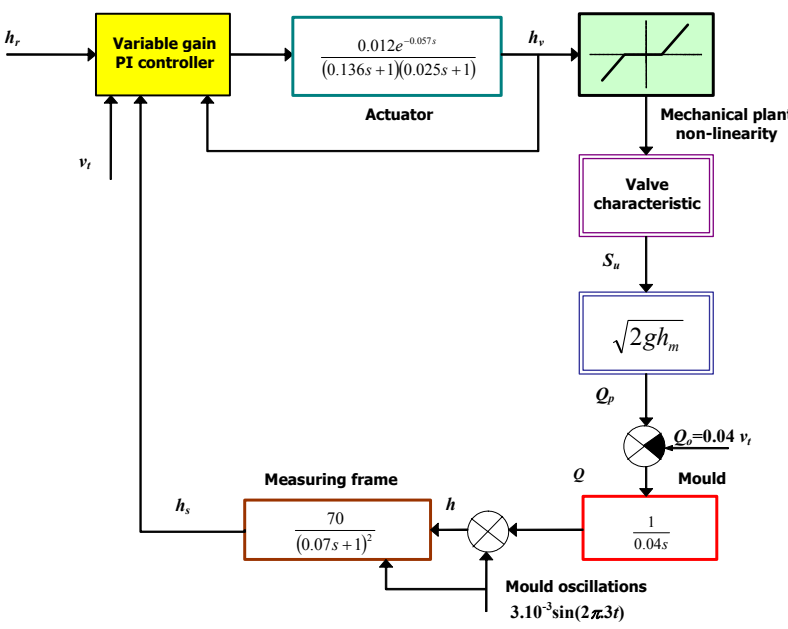

Fig. 3. Block scheme of control system structure

In Fig. 3 the block scheme of a control system is shown. It is assembled from an actuator, a valve, a mould, and a measuring frame that determines the steel level in the mould. As an actuator, an electric servo drive was used, supplied by the French firm SERT. The servo drive has the following transfer function (VUHZ, 1996; SERT, 1987):

$$
G_{\text {servo }}(s)=\frac{0.012 e^{-0.057 s}}{(0.136 s+1)(0.025 s+1)}
$$

The transfer function was given by the drive manufacturer at the time of concluding the contract. The parameters have changed a bit lately, but the given transfer function always satisfied the needs during simulation and practical tests on the actual equipment.
Fig. 3 shows that the source of very strong nonlinearity which is difficult to determine and is the transition of movement form the servo drive to the valve motion. The mechanical construction was in this case very poor and in addition its condition suffered by an unpleasant environmental impact. The mechanical maintenance was unsatisfactory as well. All these factors added to undefined and large nonlinear behaviour of the controlled system. The mechanical defect and therefore emerging nonlinearity caused an undesired system response to a control action. The elimination of the non-linearity was consequently one of the main aims of the work. The non-linearity was tried to shift off in two ways: mechanical repairs or some tune ups, and by special control algorithms that itself eliminates different kinds of non-linearities.

Another type of non-linearity emerging in the system was the stopper characteristic, see Fig. 4

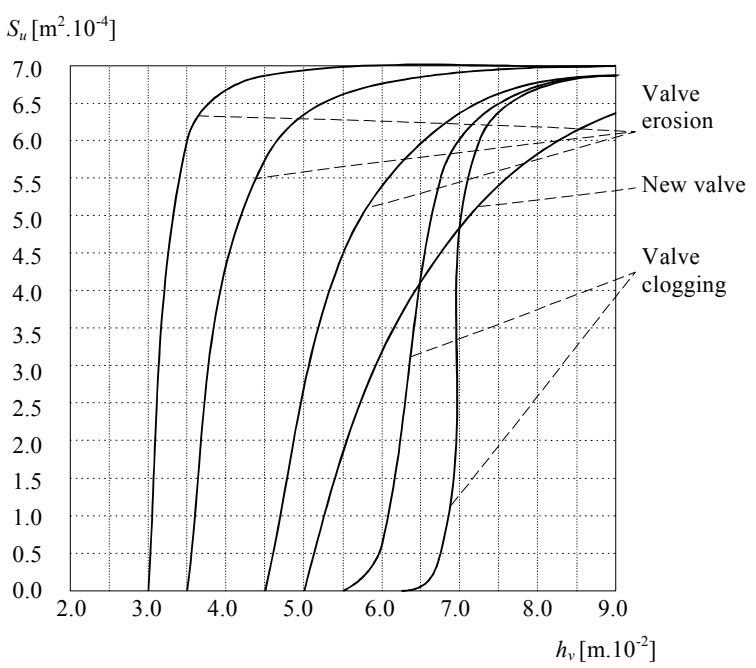

Fig. 4. Stopper characteristics including all types of disturbances.

A stopper plug, stopper saddle, and stopper rod are made of high temperature ceramics to resist the high temperature aggressive molten steel for longer periods. During the continuous casting process two types of disturbances must be taken into account. The first one is so called ,valve clogging“ that is caused by material and mixtures sticking both on the stopper saddle and stopper plug. Valve clogging shifts the stopper characteristics to the right, making it steeper in the central part and flatter in the beginning and in the end.

The second error state is called „valve erosion“, which is caused by the ceramic material erosion. In this case, the stopper plug diameter decreases and/or the stopper saddle diameter increases. The stopper characteristic then shifts to the left having its beginning and central part very steep and upper part quite flat. Both disturbances are highly undesired because they create generally more sensitive behaviour of the stopper in its operational part of the 
characteristics and a less sensitive response in the limit cases.

The valve erosion is a relatively slow process and therefore in the case of applying a robust controller the characteristic changes are usually handled satisfactorily. However, in the case of valve clogging there is usually a problem. As the valve erosion, valve clogging is a relatively slow process, but, once the sticking layer is thick enough it peels off causing a sudden stopper characteristic change.

Another non-linearity appearing in the controlled subsystem is a flow velocity from the tundish that can be described as a square root

$$
v_{m}=\sqrt{2 g h_{m}}
$$

The mould itself behaves as a simple integral system with the integral time constant $0.04 \mathrm{~s}$ (VUHZ, 1996).

$$
G_{\text {mould }}(s)=\frac{1}{0.04 s}
$$

There are many other error signals, one of which, is easily determinable. It is a periodical signal added at the point of a measuring sensor and is given by the mould oscillation.

$$
h_{\text {osc }}(t)=3 \cdot 10^{-3} \sin (6 \pi t)
$$

The whole mould is made to swing by a cam with an angle frequency $6 \pi$ and magnitude $3.10^{-3} \mathrm{~m}$. The aim of making the mould swing is to prevent a slab from sticking to the inner sides of the mould.

At the beginning of work the oscillation signal was registered by the measuring frame and processed together with level information as its integral part. Therefore different filters inside the control unit were used to strive the oscillation from the signal. The problem of signal filtering itself was lately solved on in cooperation with a Czech company. The result was a new generation of intelligent mould level sensor. The new sensor itself filters out the perturbing periodic signal. In order to preserve the high responsibility of the sensor it was impossible to use a standard filtration algorithm that will always incorporate certain inertia. The team of developers decided in this special case to chose the very simple and effective method of inverse signal subtraction in which case the sensor dynamic wasn't affected. The measuring frame dynamic is described by transfer function

$$
G_{\text {frame }}(s)=\frac{70}{(0.07 s+1)^{2}}
$$

\section{CONTROLLER DESIGN}

According to the system structure, two controller versions were designed that were subsequently tested. The first type of the controller was P-PI or PI-
PI controller that means classical controllers with a switched structure. The structure of the controller depends on the control deviation amplitude. In order to eliminate huge deviations quickly the controller switches automatically its inner structure to proportional. For small deviations the structure is PI. The mathematical controller definition then looks like:

if $e_{\min } \leq e[k T] \leq e_{\max }$ where $e_{\min }=-2.5 \mathrm{~mm}$ and $e_{\max }$ $=2.0 \mathrm{~mm}$

then PI controller has the following parameters:

$$
k_{r}=0.4 ; T_{I}=1.8 \mathrm{~s}
$$

else (that means when $e[k T] \leq e_{\min }$ OR $e[k T] \geq e_{\max }$ ) then $\mathrm{P}$ controller has the following parameter:

$$
k_{r}=0.4
$$

All the controller algorithms were created in velocity form, which is more suitable for computer implementation than algorithms in position form. Hence the system never gets out of control during the closed loop disconnection.

The discrete PI controller in velocity form:

$$
u[k T]=u[(k-1) T]+k_{r}\left(1+\frac{T}{T_{I}}\right) e[k T]-k_{r} e[(k-1) T]
$$

and the $\mathrm{P}$ controller:

$$
u[k T]=k_{r} e[k T]
$$

The software PI controller, implemented on a personal computer that is equipped with a data acquisition card, has the nonlinear static characteristic - the saturation type. The analogue amplifier working range $\left( \pm U_{\text {sup }}\right)$ causes the nonlinearity. This phenomenon can result in overshoot or undershoot during a control process. To alleviate the undesired behaviour it is required to switch the controller's integral part off when the saturation occurs (anti-windup variation of PI controller). After the disappearance of the saturation, the integral part can be switched back on again.

After computer simulation and practical verification on a physical model, a problem with the algorithm appeared. When the initial state of the stopper was in an extreme position which the controller evaluated as a large deviation and switched its internal structure to $\mathrm{P}$ type, it wasn't able to move the actuator to a required position. This happened since the stopper was beyond the working characteristic. Therefore the final version of controller with switched structure was PI-PI with different parameters for each PI controller.

The practical tests on the physical model proved that during the structure switch, certain, not negligible, oscillations or swings appeared.

Later development led to a continuous change of controller gain according to the deviation amplitude. 
The gain dependency on the deviation is given by the equation

$$
k_{r}(e)=k_{r 0}\left(1+\delta|e|^{n}\right)
$$

For $n=1$ to 4 . The value of $k_{r 0}$, the value of coefficients $\delta$ and the degree $n$ were determined experimentally on the computer and the physical plant and at last they were verified on the physical plant in the steelworks. The optimal values seem to be:

$$
k_{r 0}=0.4 ; \delta=0.2 ; n=2
$$

\section{CONTROLLER TUNING}

Once the model of the system was assembled, the presented analogue controller had to tune. According to Fig. 3 the plant transfer function has a form

$$
\begin{aligned}
& G_{S}(s) \frac{0.012 e^{-0.057 s}}{(0.136 s+1)(0.025 s+1)} \\
& \frac{1}{0.04 s} \cdot \frac{70}{(0.07 s+1)^{2}} \\
& \text { mould frame }
\end{aligned}
$$

For simplicity the mechanical non-linearity of the plant was ignored, the nonlinear valve characteristic was linearised, and the steel level in the mould was supposed constant. In order to avoid high order of the inertia, only the first order was considered. All the simplifications taken into account only led to orientation results, which are, however, sufficient due to the impossibility of obtaining the exact description of the system.

The mould steel level will be supposed to be 0.4 meters, which is the average value during a normal casting operation. The transfer function (9) can then be written as

$$
\begin{gathered}
G_{S}(s)=\frac{5.883 e^{-0.057 s}}{s(\underbrace{0.136}_{T_{1}} s+1)(\underbrace{0.025}_{T_{2}} s+1)(\underbrace{0.07 s+1)^{2}}_{T_{3}}} \approx \\
\approx \frac{5.883}{s(\underbrace{0.358}_{T_{1}+T_{2}+2 T_{3}+T_{d}} s+1)}
\end{gathered}
$$

A use of the substitute time constant instead of several time constants for each inertia element is undesirable from the tuning point of view. On the other hand, the substitution of the time delay by an inertial element is desirable and therefore we can expect that the combination of above mentioned substitutions is admissible.
Because the symmetric optimum method gives a result with a big overshoot (more then $40 \%$ ), and because a digital controller instead of an analogue, was used, the possibility of increasing of time constant $T_{I}^{*}$ and eventually decreasing the gain $k_{r}^{*}$ was taken into account.

\section{NON-LINEARITY ALLEVIATION}

The casting equipment in the steelworks, that had to be controlled, displayed a number of non-linearities which originated both from the mechanical elements and the technical condition of the facility. Friction and a backlash belonged amongst the strongest nonlinearities that considerably affected the controlled system. Mechanical junctions between an actuator and a stopper in the tundish was the cause. Their exact identification, however, was impossible because the following two reasons:

1. Each casting stream used a different actuator and the conditions of respective actuating mechanisms were different as well.

2. The casting equipment was granted for the trial of an algorithm, not for a deeper experiment for identification of exact parameters of the named non-linearities.

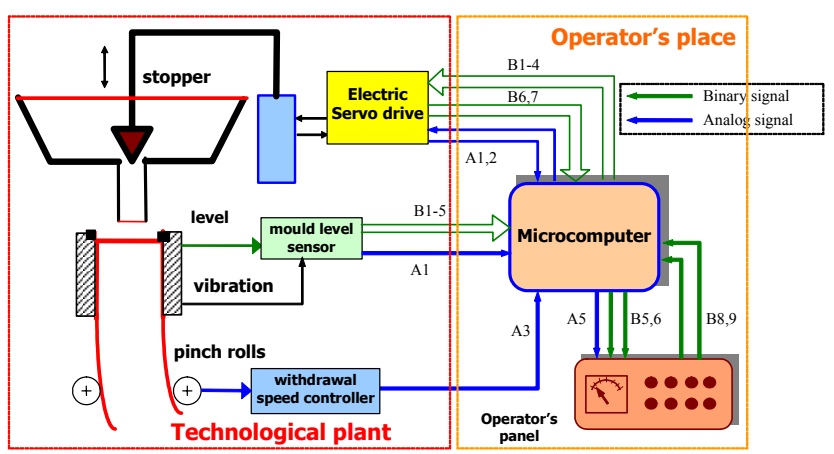

Fig. 5. Implementation of mould level control system as a real time computer system

The controlled system, of course, contained other non-linearities, that were determined exactly. The non-linearities emerge from Fig. 2. They are namely:

- the stopper nonlinear characteristic

- a liquid steel emission speed from the tundish

These non-linearities luckily don't produce a limit cycle in the controlled system that results in periodical changes of the liquid steel level in the mould. The situation is nicely illustrated in Fig.10 and Fig 11.

In the next figures (see Fig. 6, and Fig. 7) there are simulation models of the mould level control for the continuous steel casting on the simulation program SIPRO (Farana, 1996) and the next one is the result of verification of the typical simulation control task. 


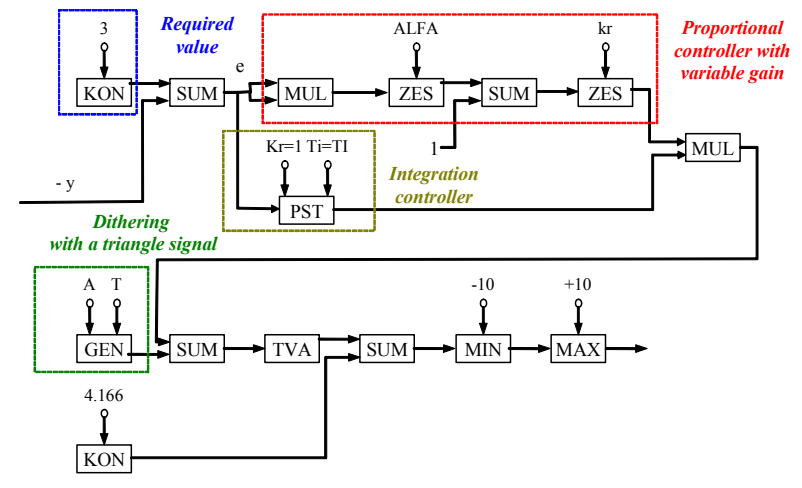

Fig. 6. Graphic form of SIPRO programme scheme of the whole mould level control system

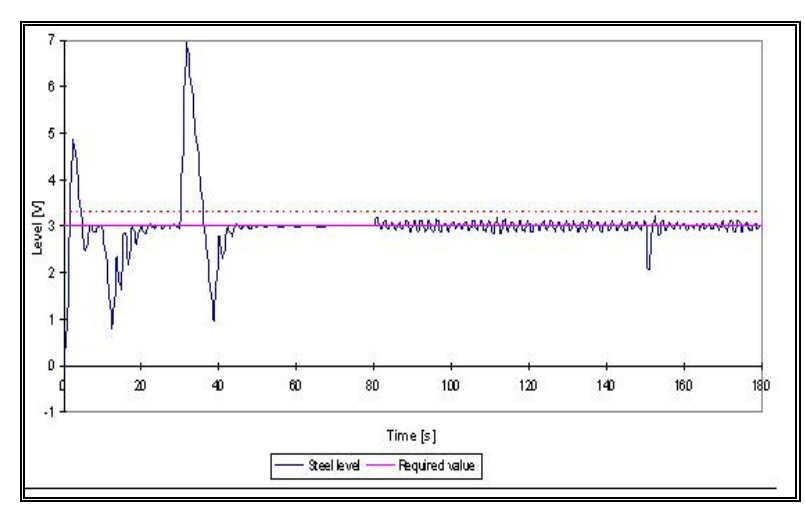

Fig. 7. Time chart of level control on SIPRO program environment with typical disturbances (hysteresis of stopper, valve clogging and erosion, ...)

Although a nonlinear behaviour in a control system may be acceptable, it is, on the whole, undesirable since it can cause performance degradation and even instability. As was described above, the system had strong non-linear characteristics. In order to alleviate the problems the principle of signal dithering was used (Cook, 1994; Wierzbicki, 1965), that makes non-linear elements behave more approximately linear in effect, by causing the operating point to sweep repeatedly over a certain range around its nominal position, on a much shorter timescale than that of the system dynamics. This is done by injecting another external signal, called dithering signal, into the system just ahead of the non-linearity.

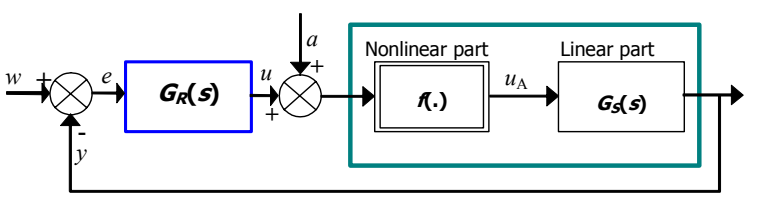

Fig. 8. Control system with dithering signal injection.

Generally many different dithering signals $a(t)$ can be applied but just two were considered for simplicity. The first is a sinusoidal dithering signal of high frequency

$$
a(t)=A \sin \left(\frac{2 \pi t}{T_{0}}\right)
$$

where the period $T_{0}$ is chosen so small that the angular frequency $2 \pi / T_{0}$ is much greater than any other involved in the operation of the system. In this case, the effective value of $u$ is its average over a complete period of the dither oscillation, namely

$$
\bar{u}_{A}=\frac{1}{2 \pi} \int_{0}^{2 \pi} f(u+A \sin \theta) d \theta
$$

The other type of signal is a triangular-wave dithering signal or „saw-tooth signal“ illustrated in Fig. 6, where $a(t)$ varies linearly with respect to time from $-A$ to $A$, and then back again.

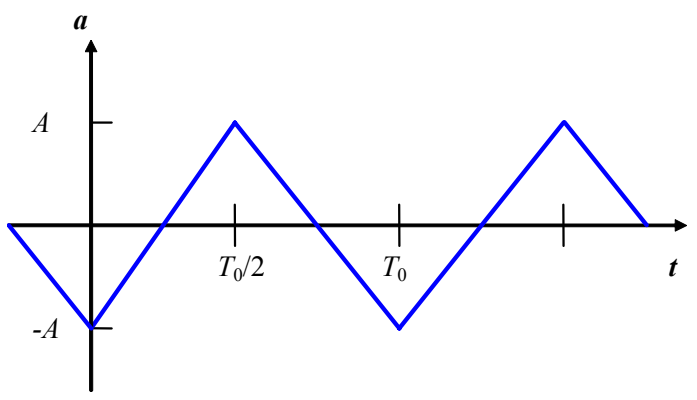

Fig. 9. Saw-tooth signal.

Because of the constancy of $\dot{a}$ on each upward and downward sweep, averaging over $t$ is equivalent to averaging over $a$, and so the effective value of $\bar{u}_{A}$ becomes

$$
\bar{u}_{A}=\frac{1}{2 A} \int_{-A}^{A} f(u+a) d a
$$

which is independent of the speed at which a actually changes. Thus, it is not in fact necessary that the time taken by the two linear sections of the oscillation should be the same, provided that the total period $T_{0}$ is small enough, in the same sense as before. However, if $f($.$) is multi-valued, the upward and$ downward averages may be different, and is given by their weighted mean value, with weighting factors proportional to the duration of corresponding sweeps. Because the $f($.$) is not known, the gain dependency$ on the deviation and dithering signal cannot be described analytically.

In Fig. 10 and Fig. 11 there are results of the mould level control system as a real time computer system. Industrial controller on PC form and the created program module make possible following choice values (required values of levels - controlled steel flow and referential flows, true position of action servo driver, action values), shifting from manual operating on an automatic regime, change of all adjustable parameters of controllers, inclusive sampling periods in both algorithms and dithering. 


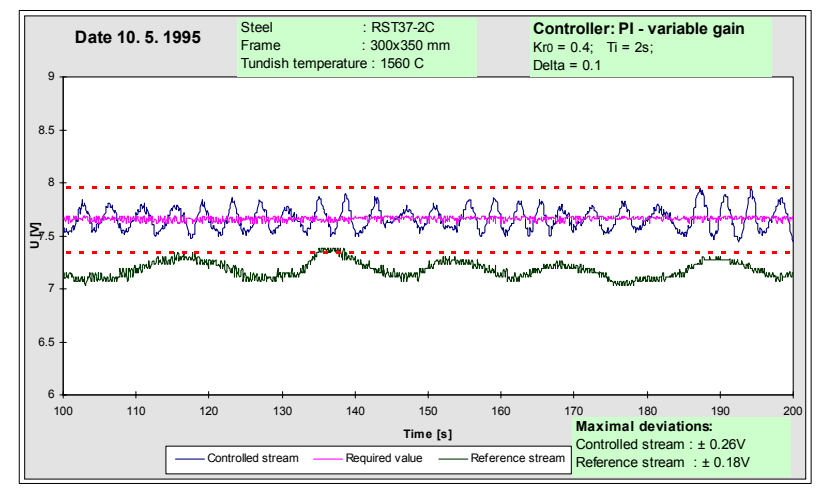

Fig. 10. PI Controller without saw-tooth dithering

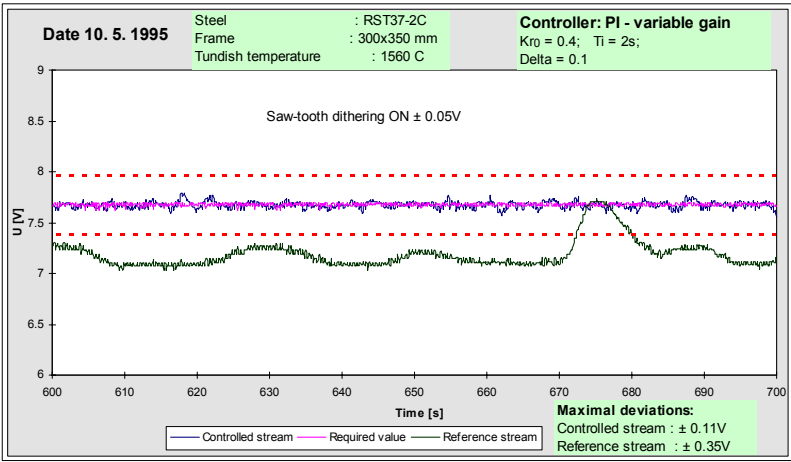

Fig. 11. PI Controller with saw-tooth dithering.

Experimental industrial verification proved that the suggested algorithm is robust enough, the quality of steel level stabilization in comparison with referential steel stream is much better and using correction vibrating signal (dithering) is considerably better.

The required parameters of control quality, i.e. acceptable fluctuation of steel level in the range \pm 3 $\mathrm{mm}$ have been achieved and at defined conditions of clearance in mechanical parts servo - drive and shoulders even better accuracy can be achieved $( \pm 1$ $\mathrm{mm})$.

The designed control algorithms make possible correct response to specified technological sequence cases, whereas they had no influence on the technological plant. There was the possibility of give a recommendation concerning technological discipline and maintaining, which has a huge influence on total quality of final products.

\section{CONCLUSIONS}

Basically, the structure of a modern mould level control of input flow comprises the following components (Kačmář, 1997):

- Level measuring equipment (sensors and electronic measuring devices)

- Process control equipment (closed loop control, sequence control and monitoring equipment)

- Electrical or electro-hydraulic servo drive

- Mechanical control equipment (stopper or gate valve)
The design and validation of the new robust algorithms under the industrial conditions of Czech Steelworks has shown that "good automatic control theory" with experimental verification is the way for the practical implementation of new trends in control design.

\section{ACKNOWLEDGEMENT}

The presented results have been obtained with the support of the Czech Ministry of Education, Youth and Sports, during completing of research project MSM 272300012.

\section{REFERENCES}

Balátě, J. (2003). Automatické ř́zení. (Automatic control). 1. edit. BEN - technical literature publishing, Prague. ISBN 80-7300-020-2.

Böcher, G., Obermann, R. and Krüger, G. (1993). Slab quality improvement by means of advanced mould level control systems. Metallurgical Plant and Technology International No.3/1993, 50-55.

Cook, P.A. (1994). Nonlinear Dynamical Systems. Prentice Hall, UK.

Farana, R. (1996). Simulační program SIPRO 3.4. Uživatelská př́ručka (Simulation Program SIPRO 3.4. User's Guide). KAKI, Ostrava.

Goodwin, G. C., Graebe, S. F., Saldago, M.E. (2001) Control System Design. Prentice Hall, Upper Saddle River, New Jersey.

Jin-Luh Su, Wen-Hsien Wang, and Cheng-Hsiung Hung (1994). The application of an automatic casting system at CSC's No. 3 bloom caster. Metallurgical Plant and Technology International, No.3/1994, 88-96.

Kačmář, D. (1997). Mould level control during continuous steel casting. Dissertation thesis. VŠB-TUO, Ostrava.

Sadao, K., Asayoshi, K., Yukihiro, S., and Hirotaka, M. (1992). A Real-time Expert system Applied to the Mould Bath Level Control of Continuous Caster. The Sumitomo Search 50, 51-58.

SERT (1987). Electromechanical cylinder documentation. SERT, Lyon France.

Šulc, B.,Vítečková, M. (2004). Teorie a praxe návrhu regulačních obvodi̊ (Theory and praxis of control system design). CTU Publishing House, Prague. ISBN 80-01-03007-5.

VÚHŽ (1996). Electromagnetic mould level detector. Production leaflets. VÚHŽ Institute, Dobrá u Frýdku Místku.

Wierzbicki, A. (1965). Korekcja układow regulacji przekaźnikowej. Archiwum Automatyki i telemechaniki. 10, volume 2, 1965, 129-147. 